\title{
The effect of underlayers on the reversal of perpendicurlarly magnetized multilayer thin films for magnetic micro- and nanoparticles
}

\author{
T. Vemulkar, ${ }^{1, \text { a) }}$ R. Mansell, ${ }^{1}$ D. C. M. C. Petit, ${ }^{1}$ R. P. Cowburn, ${ }^{1}$ and M. S. Lesniak ${ }^{2}$ \\ ${ }^{1)}$ Cavendish Laboratory, University of Cambridge, JJ Thomson Avenue, Cambridge CBз OHE, \\ $U K$ \\ 2) Feinberg School of Medicine, Northwestern University, Chicago, IL 60611, USA
}

Perpendicularly magnetized microparticles offer the ability to locally apply high torques on soft matter under an applied magnetic field. These particles are engineered to have a zero remanence magnetic configuration via synthetic antiferromagetic coupling using a Ru coupling interlayer. The flexibility offered by the top down thin film fabrication process in a $\mathrm{CoFeB} / \mathrm{Pt}$ perpendicular thin film is demonstrated by using the $\mathrm{Pt}$ interlayer thicknesses in a $\mathrm{Pt} / \mathrm{Ru} / \mathrm{Pt}$ antiferromagnetic coupling multilayer to tune the applied magnetic field value of the easy axis spin-flip transition to saturation and hence, the field value at which the magnetic particles are magnetically activated via a distinct transition to saturation. The importance of a Ta buffer layer on the magnetic behaviour of the stack is shown. While Au capping layers are desirable for biotechnology applications, we demonstrate that they can drastically change the nucleation and propagation of domains in the film, thereby altering the reversal behaviour of the thin film. The effect of Au underlayers on a multilayer thin film composed of repeated motifs of a synthetic antiferromagnetic building block is also investigated.

Magnetic particles in time-varying magnetic fields have been used to locally exert mechanical forces on soft matter for a variety of applications ${ }^{1,2}$. In particular, the transduction of energy from an applied magnetic field in the form of a local mechanical torque has garnered interest $^{9}$ for the actuation of magnetorheological elastomers ${ }^{4}$, the manipulation of cells and cellular components ${ }^{5}$, and conducting microrheology on soft matter $^{6}$. It has been recently demonstrated that a new class of planar magnetic microparticles can be fabricated, where the magnetization is perpendicular to the plane of the microdisc ${ }^{10,11}$. This magnetic particle architecture is interesting because it offers a strong uniaxial anisotropy in the surface normal direction of the microdisc. The strong perpendicular magnetic anisotropy (PMA) offers a means to actuate these magnetic particles with a well defined torque that can be tuned in magnitude via engineering the interfacial PMA. This would allow microdiscs with different magnitudes of PMA to transduce different torques locally in the same applied field. Further, the uniaxial PMA results in control over the orientation of the planar surface of the particle in a fluid environment, which is a property not available in other micro and nanoparticle systems and is exciting for optical reflection based sensor applications ${ }^{12}$.

Here we discuss the optimization of the magnetic properties of the perpendicular thin films used in the fabrication of PMA magnetic micro and nanoparticles. The magnetic system investigated is a $\mathrm{CoFeB} / \mathrm{Pt}$ heterostructure where the PMA arises from spin-orbit coupling effects at the interface between the $\mathrm{CoFeB}$ and $\mathrm{Pt}$, and is maximum for a Pt (111) texturing ${ }^{7}$. These films are characterized by sharp magnetic switching to saturation via rapid domain expansion due to the strong PMA. Further, adjacent $\mathrm{CoFeB}$ layers are antiferromagnetically (AF)

a) Electronic mail: tv243@cam.ac.uk coupled via Ruderman-Kittel-Kasuya-Yoshida (RKKY) interactions using a $\mathrm{Pt} / \mathrm{Ru} / \mathrm{Pt}$ interlayer $\mathrm{stack}^{8}$ in order to fabricate a thin film with a zero net magnetization remanent state. The sharp switch to full magnetization, which is preserved in measurements in fluid, is a property that is not observed in other systems of magnetic micro and nanoparticles and may find use in cytommetry and magnetic sorting applications in microfluidics ${ }^{13}$. The zero magnetization remanent state in the film ensures a zero net moment in the magnetic particles and is crucial for fluid based applications where magnetic agglomeration via interparticle dipolar interactions is undesirable.

The building block for such thin films is described by the schematic in figure 1 (a), and the moment and the anisotropy of the thin film dictates the strength of the torque that can be transduced from the applied magnetic field for PMA based particles. In order to increase the moment of the thin film, the basic building block is stacked repeatedly as shown in figure 1 (b). Increasing the moment in the thin film leads to a higher saturated magnetic moment per magnetic particle in order to transduce higher torque from a magnetic field. The magnetic properties of the basic building block are also tunable. The magnitude of the PMA can be tuned by the thick-

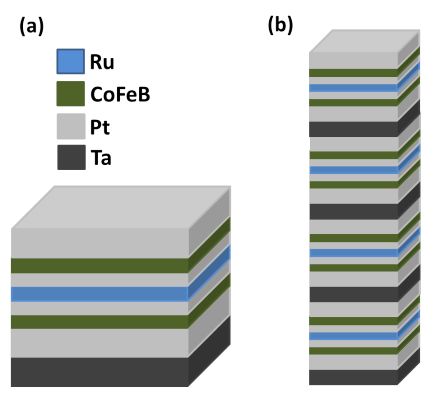

FIG. 1. (a) Schematic of the fundamental building block thin film structure. (b) A high moment thin film structure where the fundamental building block has been repeated 4 times. 
ness of the CoFeB layers. In addition to stabilizing the PMA of the second magnetic layer ${ }^{14}$, the thickness of $\mathrm{Pt}$ layers in the $\mathrm{Pt} / \mathrm{Ru} / \mathrm{Pt}$ interlayer $\mathrm{AF}$ coupling stack can be varied in order to tune the strength of the AF RKKY coupling ${ }^{8}$. The strength of the AF coupling is is measured by a shift in the minor loop of the magnetic transition $^{14}$, and is represented by a coupling field $H_{J}$.

Figure 2 (a) shows the hysteresis major and minor loops for a series of samples described by $\mathrm{Ta}(2) / \mathrm{Pt}(2) / \mathrm{CoFeB}(0.5) / \quad P t\left(t_{P t}\right) / R u(0.9) / P t\left(t_{P t}\right) /$ $\mathrm{CoFeB}(0.5) / \mathrm{Pt}(2)$. Figure 2 (b) shows the variation in the effective coupling field as a function of the thickness of the Pt layers on either side of the $\mathrm{Ru}$, for thin films with three separate values of $C o F e B$ thickness described by $\mathrm{Ta}(2) / \mathrm{Pt}(2) / \mathrm{CoFeB}\left(t_{\mathrm{CoFeB}}\right) / \mathrm{Pt}(t) / R u(0.9) / \mathrm{Pt}(t) /$ $\mathrm{CoFeB}\left(t_{\mathrm{CoFeB}}\right) / \mathrm{Pt}(2)$. The variation in the coupling strength is exponential ${ }^{8}$, of the form $H_{J}=A e^{-\frac{t_{P t}}{t_{d}}}$. The characteristic decay length of the coupling field is unchanged with CoFeB thickness with $t_{d} \approx 0.19$ $\mathrm{nm}$. The amplitude of the exponential decay function decreases with decreasing the $\mathrm{CoFeB}$ thickness, with $A \approx 10.6,8.2$ and $5.9 \mathrm{kOe}$ for $t_{C o F e B}=0.5,0.8$ and $1.0 \mathrm{~nm}$ respectively as expected. The amplitude of this exponential decay scales with the CoFeB thickness ${ }^{14}$ since the coupling field varies as $H_{J}=\frac{J}{M_{S} t_{C o F e B}}$, where $J$ and $M_{S}$ are the interlayer coupling energy per unit area and the saturation magnetization of the magnetic layers respectively.

We next study the effect of a Ta buffer layer on the magnetic behaviour of a single AF coupled building block. The magnetic behavior of PMA materials is extremely sensitive to substrate topography such as roughness and defect density ${ }^{18}$. In the $\mathrm{CoFeB} / \mathrm{Pt}$ multilayers used throughout this work, the buffer layer consists of a Ta smoothing layer, and a $\mathrm{Pt}$ seed layer. The Pt seed layer is a strong determinant of the magnitude of the PMA in the system. The PMA in the $\mathrm{CoFeB} / \mathrm{Pt}$ system arises largely from the spin-orbit coupling between the $\mathrm{Co}$ and $\mathrm{Pt}$ atoms at the layer interfaces, and the magnitude of the PMA is significantly affected by the interlayer roughness, as well as the texturing of the $\mathrm{Pt}$ layer. A Pt(111) texturing provides the largest PMA for the $\mathrm{Co} / \mathrm{Pt}$ system $^{7}$ (and hence the $\mathrm{CoFeB} / \mathrm{Pt}$ system) and the $\mathrm{Ta}$ promotes the growth of the Pt (111) texture ${ }^{19}$. Figure 3 shows major and minor polar MOKE hysteresis loops used to study the effect of the thickness of Ta on the magnetic properties of a $\mathrm{Ta}\left(t_{T a}\right) / \operatorname{Pt}(5) /(\mathrm{CoFeB}(0.5) / \operatorname{Pt}(0.7))_{2} / \mathrm{CoFeB}(0.5)$ $/ \operatorname{Pt}(0.3) / R u(0.9) / \operatorname{Pt}(0.3) /$

$(\mathrm{CoFeB}(0.5) / \mathrm{Pt}(0.7))_{2} / \mathrm{CoFeB}(0.5) / \quad \mathrm{Pt}(3) \quad$ (thicknesses in $\mathrm{nm}$ ) thin film. With $t_{T a}=0 \mathrm{~nm}$ the thin film has weak out of plane magnetic anisotropy as indicated by the slanted hysteresis loop in figure 3 (a). In figures 3 (b)-(f) we can use the relative height of the step in the Kerr signal to attribute a given transition to either the top or the bottom $\mathrm{CoFeB}$ magnetic layer since the signal from the bottom layer is more attenuated due to the skin depth of the laser. The magnetic reversal of
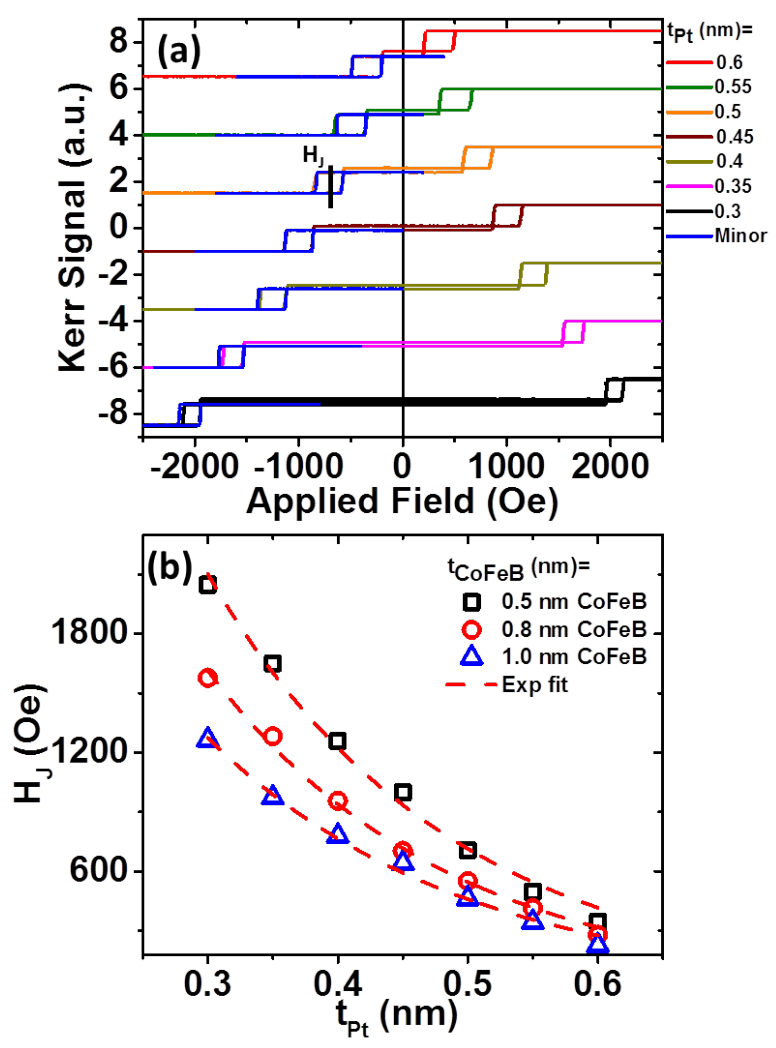

FIG. 2. (a) Polar MOKE major and minor hysteresis loops of AF coupled bilayers described by $\mathrm{Ta}(2) / \mathrm{Pt}(2) / \mathrm{CoFeB}(0.5) /$ $\mathrm{Pt}(t) / R u(0.9) / \mathrm{Pt}(t) / \mathrm{CoFeB}(0.5) / \mathrm{Pt}(2)$. (b) The effective coupling field $H_{J}$ as a function of $\mathrm{Pt}$ layer thickness for a thin films described by $\mathrm{Ta}(2) / \mathrm{Pt}(2) / \mathrm{CoFeB}\left(t_{\mathrm{CoFeB}}\right) /$ $\mathrm{Pt}(t) / R u(0.9) / \mathrm{Pt}(t) / \mathrm{CoFeB}\left(t_{\mathrm{CoFeB}}\right) / \mathrm{Pt}(2)$.

the bottom $\mathrm{CoFeB}$ layer is most significantly affected by the change in the buffer, since the transition to saturation of the top layer (corersponding to the higher jump in Kerr signal) remains sharp for all thickneses of Ta. For $t_{T a}=1$ and $2 \mathrm{~nm}$ in figure 3 (b) and (c) we observe sharp coercive switching and high anisotropy as indicated by the hard axis saturation field $H_{S A T}$ in figure $3(\mathrm{~g})$. Clearly, Ta is crucial as a smoothing layer to seed the $\operatorname{Pt}(111)$ texture. For the minor loops (blue) of thin films with $t_{T a}=3$ and $4 \mathrm{~nm}$ in figure 2 (d) and (e), the antiparallel to parallel (AP- P) transition is more slanted than the parallel to anti-parallel (P-AP) transition. This is indicative of topographical defects in the film that cause an increase in the number of sites of high local ferromagnetic coupling ${ }^{16}$. Growth defects in the thin films such as pinholes in the AF coupling layer lead to this asymmetry in the transition, since there is an increase in the number of nucleation sites for the AP-P transition. The anisotropy is also slightly reduced for these films as evidenced by the hard axis saturation fields seen in figure $3(\mathrm{~g})$. We attribute this trend to a gradual emergent texturing from an amorphous to a slightly polycrystalline state as the thickness of deposited Ta is increased, leading to a change in the roughness of the Ta layer ${ }^{17}$. When 


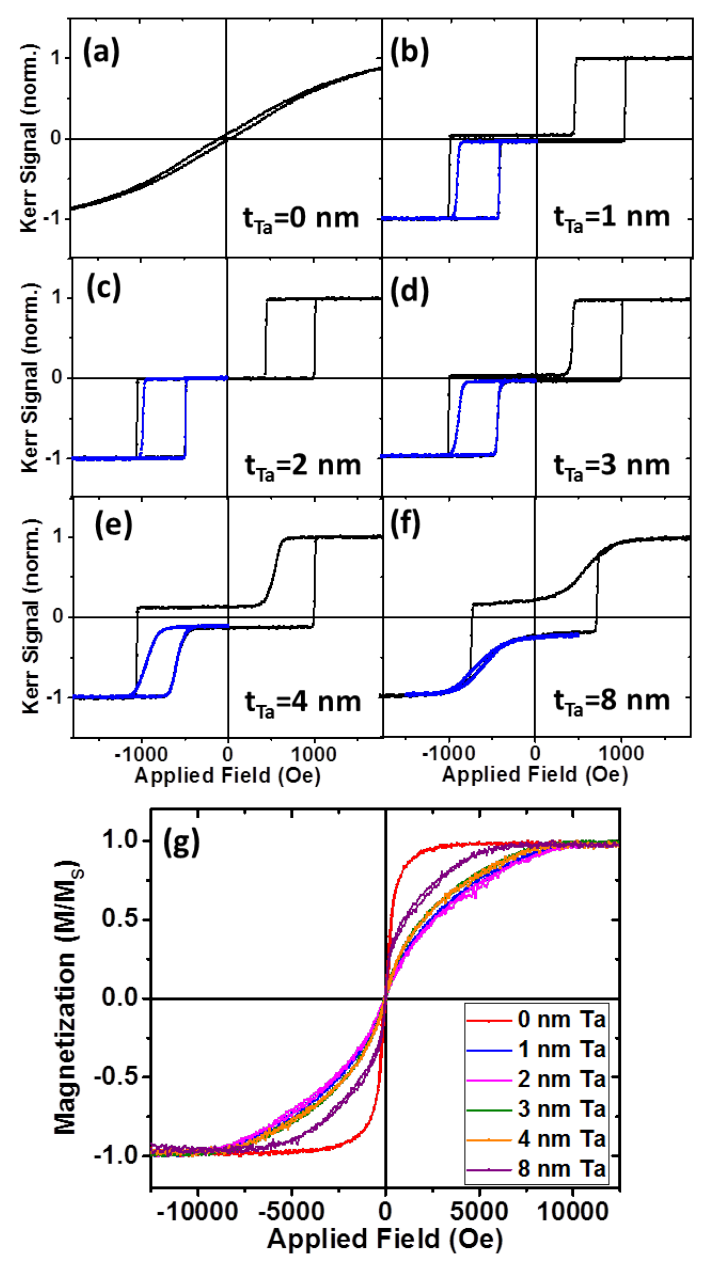

FIG. 3. Polar MOKE major (black) and minor (blue) hysteresis loops of an AF coupled bilayer described by $T a(t) / P t(6) / C o F e B(0.8) / \quad P t(0.6) / R u(0.9) / P t(0.6) /$ CoFeB(0.8)/Pt(2). (a) $t_{T a}=0 \mathrm{~nm}$. (b) $t_{T a}=1 \mathrm{~nm}$. (c) $t_{T a}=2 \mathrm{~nm}$. (d) $t_{T a}=3 \mathrm{~nm}$. (e) $t_{T a}=4 \mathrm{~nm}$. (f) $t_{T a}=8$ $\mathrm{nm}$. (g) Hard axis hysteresis loops for the samples in (a)-(f). Variation of $H_{J}$ and $H_{S A T}$ over the range of $t_{T a}$.

$t_{T a}=8 \mathrm{~nm}$ we see a significant reduction in the PMA in of the stack. The minor loop in figure 3 (e), for instance, clearly shows that the bottom CoFeB layer is the one more significantly affected by the Ta texturing since it is the layer switching during the P-AP transition. The magnetic switch in the bottom layer occurs via a multi-domain transition to saturation evident from the slanted minor hysteresis loop. We observe that the AP-P transition which corresponds to the switching of the top CoFeB layer is significantly sharper in figures 3 (d)-(f) and this suggests that the roughness of the Ta is slightly smoothened out by the layers above the bottom CoFeB layer allowing for magnetic saturation via rapid domain in the top layer. Hence, it is not simply the choice of the buffer layer, but also its thickness that can be critical to the magnetic behaviour of the multilayer thin film. Buffer layer considerations are particularly important

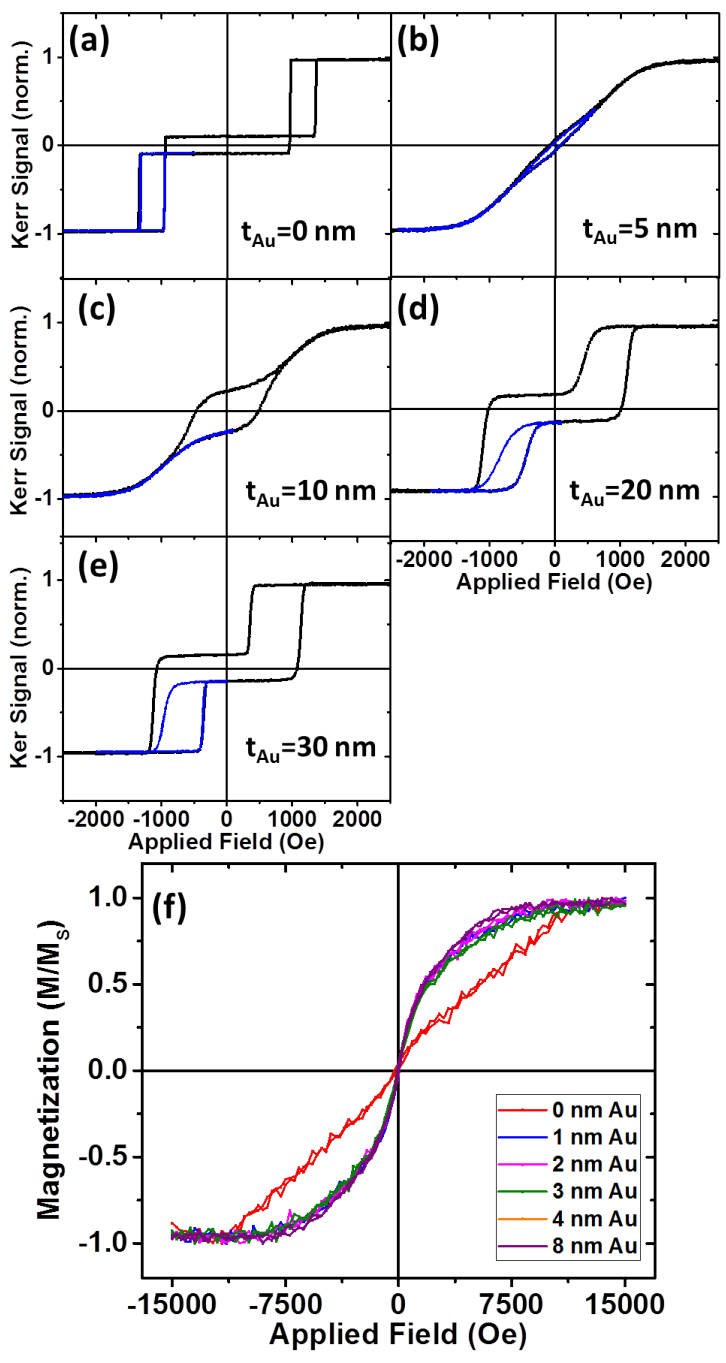

FIG. 4. Polar MOKE major (black) and minor (blue) hysteresis loops of an AF coupled bilayer described by $A u(t) / T a(2) / P t(2) / C o F e B(0.9) / P t(0.3) / R u(0.9) / P t(0.3) /$ CoFeB(0.9)/Pt(2) with (a) $t_{A u}=0 \mathrm{~nm}$. (b) $t_{A u}=5 \mathrm{~nm}$. (c) $t_{A u}=10 \mathrm{~nm}$. (d) $t_{A u}=20 \mathrm{~nm}$. (e) $t_{A u}=30 \mathrm{~nm}$. (f) Hard axis hysteresis loops for the samples in (a)-(f).

for the deposition of these multilayers on photoresist during the top down fabrication process of particles to be released in liquid.

Since the magnetic particles fabricated from these thin films are to be used in biotechnological applications, it is desirable to add Au cappping layers on either side of the particle. While the top Au cap has no effect on the magnetic properties of the thin film stack, it is clear from our work thus far on the Ta buffer, that a new underlayer material can significantly affect the magnetic properties of the thin film that is being grown. Figure 4 (a)-(e) summarises the effect on the easy axis magnetic reversal of adding an $\mathrm{Au}$ underlayer for a series of thin films described by $A u(t) / T a(2) / P t(2) / C o F e B(0.9) /$ $\operatorname{Pt}(0.3) / R u(0.9) / \operatorname{Pt}(0.3) / \quad C o F e B(0.9) / P t(2) \quad$ with $t_{A u}=0,5,10,20$ and $30 \mathrm{~nm}$ respectively. Figure 4 (f) 
summarizes the hard axis response of the films. The increased hard axis saturation fields in figure 4 (e) compared to the easy axis saturation fields in figure 4 (a)-(e) shows that the films have PMA for all $\mathrm{Au}$ underlayer thicknesses. Figure 4 (a) shows that with no $\mathrm{Au}$ buffer we observe the expected sharp coercive switching. However, figure 4 (b) and (c) show clearly that 5 and $10 \mathrm{~nm}$ of $\mathrm{Au}$ significantly affect the reversal mechanism. The slanted loops with no coercivity indicate a system with an extremely high defect population. For figure 4 (b) and (c), a shift in the minor loop is undefined and so we cannot define a coupling field $H_{J}$ for these two films, but we observe that the hysteresis loop is stretched by the effect of the AF coupling and the easy axis saturation field for both figure 4 (b) and (c) is comparable to that of the figure 4 (a). This suggests that the magnitude of the AF coupling is not significantly reduced despite the magnetic reversal being drastically changed. Figure $4(\mathrm{~d})$ and (e) show that as the $\mathrm{Au}$ thickness is increased more robust and coercive magnetic switching begins to emerge. We can now define a shift in the minor loop, and $H_{J}$ for these two films is comparable to that of figure 4 (a). From figure 4 (f), we observe that the Au underlayers cause the same reduction in the anisotropy from a hard axis saturation field $H_{S A T} \approx 11000$ Oe with no Au underlayer, to an $H_{S A T} \approx 7300 \pm 500$ Oe for the thin films grown on the various $\mathrm{Au}$ underlayers. We directly attribute this to a reduction in the magnitude of the PMA when an $\mathrm{Au}$ underlayer is used, since $H_{S A T}=H_{K}+2 H_{J}$ where $H_{K}$ is the effective perpendicular anisotropy field of the individual layers. It is also clear that the nature of domain nucleation and propagation is significantly affected by the $\mathrm{Au}$ underlayer. The transitions get sharper and more coercive with increasing Au thickness, suggesting that there is a reduction in the number of reverse domain nucleation sites, and a smaller spread in the domain nucleation fields. A Ti seed layer grown below the $\mathrm{Au}$ layer has been shown to significantly improve the texturing of $\mathrm{Au}$ films ${ }^{20}$ but this is not an option available in applications where Au capping layers are desirable.

Figure 5 (a)-(d) are AFM topography scans for Au thin films of thickness 5, 10, 20, and $30 \mathrm{~nm}$ respectively (note the difference in the scale for the height profile for each scan). Figure 5 (e) is a power spectral density (PSD) plot of the surface profiles for each of the Au thin films. It is clear from figure 5 (e) that as the $\mathrm{Au}$ thickness is increased there is a significant reduction in the rougness on the length scale of $10-25 \mathrm{~nm}$ highlighted in figure 11 (c) by a black arrow. Magnetic domain walls in perpendicular thin films with the anisotropy similar to that of the system shown here have characteristic widths on the order of tens of $\mathrm{nm}^{21}$. A high population of defects on this length scale is likely to have a prominent effect on the nucleation and pinning of the magnetic domain walls, thus affecting the magnetic reversal process ${ }^{22}$. A reduction in the texturing on this length scale leads to the sharper reversal transitions that we observe in figure 10 as $t_{A u}$ is increased from 5 to $30 \mathrm{~nm}$.

Figure 5 (f) shows the effect of a $20 \mathrm{~nm}$ buffer on a thin film where the basic building block is stacked 4 times, and is described by $[A u(20) / T a(2) / P t(2) / C o F e B(0.9)$ $/ \operatorname{Pt}(0.3) / R u(0.9) / \operatorname{Pt}(0.3) / \operatorname{CoFeB}(0.9) / \operatorname{Pt}(2)]_{4}$. The relative height of the polar MOKE Kerr signal step from conducting a series of minor loop measurements allows us to identify the transitions corresponding to each bilayer motif, and deduce an $H_{J}(X)$ for $X=1,2,3,4$, associated with each motif. Hence, we can observe how the strength of the AF coupling varies in the thin film vertically away from the Au underlayer. We see from figure 4 (f) that $H_{J}(X)$ increases away from the Au underlayer as we move up vertically in the thin film stack. We attribute this to the effect of the successive Ta smoothing layers that are deposited as a buffer for each bilayer motif. We expect the increasing strength of the AF coupling to reach a peak as $X$ increases since eventually the increasing interlayer roughness arising from increasing the stack thickness during the growth process is likely to counteract the smoothing effect of the Ta buffers between subsequent building blocks.

In conclusion, PMA magnetic particles are a new class
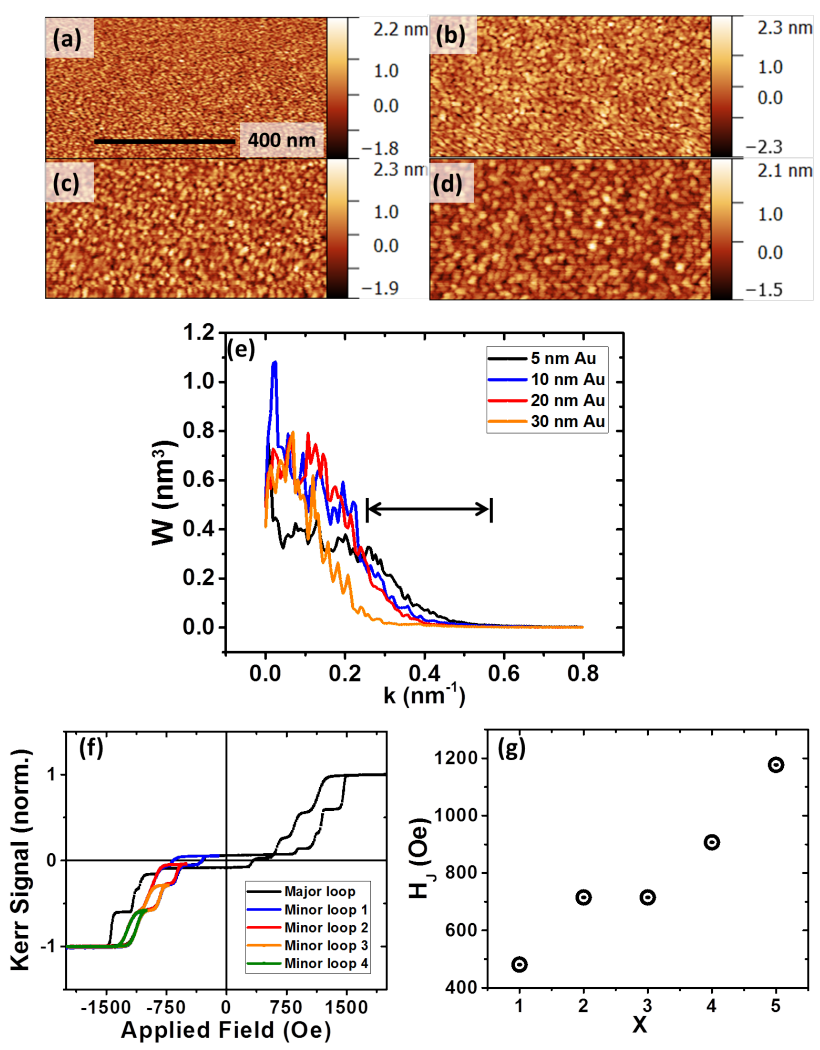

FIG. 5. AFM scans for Au layers of thickness (a) 5, (b) 10, (c) 20 and (d) $30 \mathrm{~nm}$. (e) PSD plot derived from the AFM scans in (a)-(d). (f) Polar MOKE major (black) and minor (various) hysteresis loops for a film described by $[A u(20) / T a(2) / P t(2) / C o F e B(0.9)$ $/ P t(0.3) / R u(0.9) / P t(0.3) / C o F e B(0.9) / P t(2)]_{4}$. (g) Variation of $H_{J}$ as a function of $X$ from (f). 
of magnetic micro and nanoparticle that are well suited to a variety of applications involving the transduction of mechanical torque from an applied magnetic field. The optimization of PMA thin films for the fabrication of such particles requires the precise engineering of buffers, underlayers, and interlayer thicknesses. We demonstrate here the tunability in the magnetic properties of the thin film, as well as the drastic effect of a smoothing Ta buffer, and a Au underlayer. Tuning the magnetic properties of the thin film leads to direct control of the magnetic response of the particles, and this allows for application specific design of magnetic particles relevant to fields ranging from biotechnology to soft robotics.

${ }^{1}$ Q. A. Pankhurst, N. T. K. Thanh, S. K. Jones, and J. Dobson, J. Phys. D: Appl. Phys. 42224001 (2009)

${ }^{2}$ D.-H. Kim, E. A. Rozhkova, I. V. Ulasov, S. D. Bader, T. Rajh, M. S. Lesniak, and V. Novosad, Nature Materials 9, 165 (2010)

${ }^{3}$ R. M. Erb, J. J. Martin, R. Soheilian, C. Pan, and J. R. Barber, Adv. Funct. Mater., 26: 38593880 (2016)

${ }^{4}$ J. Kim, S. E. Chung, S.-E. Choi, H. Lee, J. Kim, S. Kwon, Nat. Mater. 2011, 10, 747.

${ }^{5}$ J. Dobson, Nature Nanotechnology 3, 139 (2008)

${ }^{6}$ A. Brasovs, J. Cimurs, K. Ērglis, A. Zeltins, J.-F. Berret and A. Cēbers, Soft Matter, 11, 2563 (2015)

${ }^{7}$ M. T. Johnson, P. J. H. Bloemen, F. J. A. den Broeder, and J. J. deVries, IOP Science, Rep. Prog. Phys., 59, 1409 (1996)

${ }^{8}$ R. Lavrijsen, A. Fernández-Pacheco, D. Petit, R. Mansell, J. H. Lee, and R. P. Cowburn, Appl. Phys. Lett., 100, 052411 (2012)
${ }^{9}$ R. M. Erb, J. J. Martin, R. Soheilian, C. Pan, and J. R. Barber, Adv. Funct. Mater. (2016)

${ }^{10}$ T. Vemulkar, R. Mansell, D. C. M. C. Petit, R. P. Cowburn and M. S. Lesniak, Appl. Phys. Lett. 107, 012403 (2015)

${ }^{11}$ M. E. Muroski, R. A. Morshed, Y. Cheng, T. Vemulkar, R. Mansell, Y. Han, L. Zhang, K. S. Aboody, R. P. Cowburn, M. S. Lesniak, PLoS ONE, 11(1), e0145129 (2016)

${ }^{12}$ D. M. Bruls, T. H. Evers, J. A. H. Kahlman, P. J. W. van Lankvelt, M. Ovsyanko, E. G. M. Pelssers, J. J. H. B. Schleipen, F. K. de Theije, C. A. Verschuren, T. van der Wijk, J. B. A. van Zon, W. U. Dittmer, A. H. J. Immink, J. H. Nieuwenhuis and M. W. J. Prins, Lab on a Chip 9, 3504 (2009)

${ }^{13}$ C. W. Shields IV, C. D. Reyes, and G. P. López, Lab Chip, 2015 $15,1230(2015)$

${ }^{14}$ S. Bandiera, R. C. Sousa, S. Auffret, B. Rodmacq, and B. Dieny, Appl. Phys. Lett., 101, 072410 (2012)

${ }^{15}$ J. Moritz, F. Garcia, J. C. Toussaint, B. Dieny, J. P. Nozières, Europhys. Lett., 65, 123 (2004)

${ }^{16}$ J. H. Lee, R. Mansell, D. Petit, A. Fernández-Pacheco, R. Lavrijsen, and SPIN R. P. Cowburn, SPIN, 3, 4 (2013)

${ }^{17}$ K. Stella, D. Bürstel, S. Franzka, O. Posth and D. Diesing, J. Phys. D: Appl. Phys. 42, 135417 (2009)

${ }^{18}$ A. Paul, J. Magn. Magn. Mater., 240, 497 (2002)

${ }^{19}$ R. Law, R. Sbiaa, T. Liew and T. C. Chong, Appl. Phys. Lett. 91, 242504 (2007)

${ }^{20}$ M. Matczak, B. Szymaski, M. Urbaniak, M. Nowicki, H. Gowiski, P. Kuwik, M. Schmidt, J. Aleksiejew, J. Dubowik, and F. Stobiecki, Journ. Appl. Phys.114, 093911 (2013)

${ }^{21}$ T. A. Moore, I. M. Miron, G. Gaudin, G. Serret, S. Auffret, B. Rodmacq, A. Schuhl, S. Pizzini, J. Vogel, and M. Bonfim, Appl. Phys. Lett., 93, 262504 (2008)

${ }^{22}$ T. Vemulkar, R. Mansell, A. Fernández-Pacheco and R. P. Cowburn, Adv. Funct. Mater., 26, 26 (2016) 